\title{
Methodology of machine destruction testing
}

\author{
Vladimir Boykov ${ }^{1}$, Aleksandr Povarecho $^{1, *}$ \\ ${ }^{1}$ BNTU University of Minsk, Faculty of Mechanical Engineering, Belarus
}

\begin{abstract}
This paper presents selected problems connected with automation of procedures involved in assessment of machine degradation degree using vibration method with special emphasis on the machine state prognosis. The current knowledge of these problems is not sufficient and needs further research on data processing, analysis of efficiency of diagnostic and prognostic procedures, collection and selection of diagnostic parameters and development of automatic procedures for recognition and prognosis of a machine state. New solutions and different aspects of diagnostic prognosis based on the proposed partial procedures focus on factors determining automation of procedures for identification of technical systems states. New automated procedures for acquisition and processing of symptoms indicating the machine state provide better possibilities of control and supervision of technical systems operation and maintenance through identification of their current states, and its good prognosis.
\end{abstract}

\section{Introduction}

Increasing demand for methods and means of technical diagnostics, that is tools for assessment of technical systems degradation states, meets the needs of contemporary entrepreneurs who use a variety of technical appliances to run their businesses. Destruction processes affecting technical systems have a direct influence on their operation safety and therefore it is necessary to monitor their technical state [1,10,23]. Methods and means of modern technical diagnostics are tools for diagnosing states of technical systems which provides the basis for making operational decisions [4].

Constantly changing demands and new possibilities have generated new categories of research problems, highlighted others and disqualified some issues which have lost their importance in terms of application due to [15,22]:

- access to advanced world technologies;

- availability of the newest generation research equipment;

- possibilities of the latest computer applications in the field of hardware and software;

- access to databases, capital market and vast possibilities of cooperation.

All this have diametrically changed the views and achievements in automation of detection and control of technical object state changes by methods of technical diagnostics, especially in the field of shaping the current and predicted safety of technical objects. This provides the possibility of computerization of the processes connected with an object operation, supervision of the state changes, location of faults and minimization of damage effects and safety threats [12,21,24].

Technical diagnostics of mechanical engineering is based on utilization of information about the machine changes which enables identification of safety threats and progressing destruction of a machine throughout its life cycle. Changes of the state, reflected by methods of technical diagnostics, prevent from occurrence of causes and effects of damage $[2,3,16]$. A factor which boosts development of machine diagnostics is the responsibility of the function it performs reduction of the following threats: threat to human life and health,

\footnotetext{
*Corresponding author: POVAREKHO@bntu.by
} 
threat to natural and technical environment, threats to operation quality and economic risks $[8,20,25]$. The need to assess the technical condition of a machine results from the necessity of making decisions on the machine quality and its further operation. It can be a decision concerning its further use, taking preventive actions or altering its structure, use of technologies or maintenance methods.

This work deals with selected problems connected with the machine state control and monitoring in time, focusing the machine operation process and the final product quality shaping, diagnostic modeling and control of a machine usability in its operation and maintenance by means of prognosis methods - in terms of developing faults. Computer aided modeling of an object lifetime, degradation processes, operation states, operation control and decision support systems - are the main issues to be addressed to provide technical systems with efficient operation.

\section{Description of the machine wear process}

Occurrence of faults is one of events that accompany the process of machine operation and maintenance and determines its reliability, efficiency and the way it is operated. After being damaged a machine (assembly, component) becomes unusable. A usable machine can perform tasks it is designed with simultaneous maintenance of parameters specified in its technical documentation. Due to the impact of the environment and performance of the assigned tasks the object initial properties can be changed in the following way [26]:

- in result of irreversible aging and wear processes that take place in the machine;

- in result of occurrence of reversible processes with different intensity, caused by exceeding permissible values of one or more forcing factors;

- in a step - like manner which is manifested by a non-constant transition of one or two features beyond the limits accepted for a given machine.

The major causes of damage occurrence are classified as follows:

a) structural - faults due to design and construction errors, most often when extreme loads are neglected;

b) production related (technological) - faults which occur due to errors and imperfections of technological processes (no dimensions tolerance, insufficient surface smoothness, thermal treatment, etc.) or faults of materials used in the object or its structural components;

c) operational - faults which occur in effect of failing to observe respective rules of operation or due to the impact of external factors unexpected for the object service conditions;

d) due to aging and wear - always accompany operation of objects and are caused by irreversible changes that impair strength and cooperation of components.

In the energy-based approach, the object damage occurs under the influence of energy transmitted by it. Depending on the kind of energy prevailing in given conditions can be divided into:

a) mechanical - static stresses, creeping, fatigue, pitting, friction wear;

b) chemical - metal corrosion, rubber, paint and insulation aging, wood rotting;

c) electrical - electro-corrosion;

d) thermal - hyper melting, intensification of occurring phenomena.

The process of wear and degradation of functional qualities is not homogenous and can be divided into four physically different degradation processes. In the first place, it is fatigue wear that should be mentioned as it leads to loss of cohesion of the structure components and is caused by too high dynamic stresses - oscillatory, that is vibrations. The second is friction wear in all forms which occurs in kinematic pairs and leads to losses in mass - backlashes. Rheological properties of construction materials cause the third degradation process involving creeping (floating) leading to changes in the size and shape 
of the structure stressed elements. The fourth way of erosive wear of machine components involves physical (cavitation) or chemical (corrosion) degradation resulting in the material mass loss.

This work takes advantage of the basic assumption, already proven in many studies, that knowledge of relations between the machine model described by parameters: $\mathrm{m}, \mathrm{k}, \mathrm{c}(\mathrm{m}-$ mass, $\mathrm{k}$ - stiffness, $\mathrm{c}$ - damping), and quantities a, $\mathrm{v}, \mathrm{x}$ ( $\mathrm{a}$ - acceleration, $\mathrm{v}$ - velocity, $\mathrm{x}-$ displacement) describing the vibration process provides big possibilities and vast applications of vibration-based diagnostics.

Tribology deals with all the phenomena connected with a machine wear. It assumes on proportionality of vibrations $\mathrm{D}$ to the wear processes advancement [4,5]. Creation of a mathematical model of a machine about all the acoustic and wear phenomena is a tribology approach. Advanced wear of a machine - tribological $E_{z}$ is best measured by energy lost for irreversible wear processes - tribological. Having in mind that a new machine (with no wear) is already characterized by some level of rated vibration $D_{n}$, the constitutive relations of diagnostics can be expressed as follows:

$$
\mathbf{D}=\mathbf{D}_{\mathbf{n}}+\gamma \mathbf{E}_{\mathbf{z}}
$$

where: $D$ - amplitude measurement of vibration - e.g. acceleration, velocity etc.; $D_{n}$ level of rated vibration of a new machine, $E_{z}$ - energy lost for irreversible processes of wear, $y$ - coefficient of tribovibroacoustic lossiness (TWA) depicting correlation between tribological processes and vibrations [4].

Knowing the machine mechanical efficiency $\eta$ it is possible to estimate lost energy $E_{z}$, as:

$$
\eta=\frac{N_{u \dot{z}}}{N_{z a s}}=\frac{N_{z a s}-N_{z}}{N_{z a s}}=\frac{E_{z a s}-E_{z}}{E_{z a s}}
$$

where: $N_{U Z}-$ is useful power, $N_{z a s}$ - feed power, $E$ - respective energies.

Tribological processes which most frequently accompany machine motion include fatigue and friction, whereas their energy is generated proportionally to the fatigue crack length $\boldsymbol{l}$ and the volume of the worn material $U$. Focusing on the two phenomena it can be accepted:

$$
E_{z}=E_{\text {tarcia }}+E_{\text {zmęmęczen }}+\ldots \cong a l+b U
$$

whereas: $a$ and $b$ - certain coefficients of proportionality, 1 - computed from Paris formula [12], $U$ - from Archard formula [17].

Computing fatigue crack length from Kocańda formula [8] is:

$$
l=C_{3}\left(\sigma_{0}+\sigma_{a}\right)^{n} N=C_{3}\left(\sigma_{0}+\sigma_{a}\right)^{n} \cdot f \cdot \theta=C_{3}\left(\sigma_{0}+e D\right)^{n} \cdot f \cdot \theta
$$

where: e - coefficient of vibration proportionality, $D$ - to variable stresses $\sigma a, \theta$ - time of their operation, $f$ - vibration frequency.

When dynamic component of thrust $p=p_{0}+g D$ with coefficient of proportionality to vibration amplitude $g$ is introduced into Archard [17] equation we receive:

$$
U=\frac{k_{0} \cdot v \cdot \theta}{R_{e}}\left(p_{0}+g D\right)
$$

Substituting $E_{z}$ to expression (3) describing energy of wear processes, and this in turn to constitutive relation (1) we receive: 


$$
\begin{aligned}
& D=D_{n}+\gamma\left[a C_{3}\left(\sigma_{0}+e D\right)^{n} \cdot f+\frac{b k_{0} v}{R_{e}}\left(p_{0}+g D\right)\right] \cdot \theta=D_{n}+\gamma N_{z} \theta \\
& N_{z}=a C_{3}\left(\sigma_{0}+e D\right)^{n} \cdot f+\frac{b k_{0} v}{R_{e}}\left(p_{0}+g D\right)
\end{aligned}
$$

into which the power of wear processes $\mathrm{NZ}=E_{z} / \Theta$ depending on vibration amplitude $D$ is introduced.

Using these dependencies for final determination of the machine vibration amplitude in a function of its operation time we can find:

$$
D=\frac{\gamma N_{z}^{0} \cdot \theta}{1-\left.\gamma \theta \frac{d N_{z}}{d D}\right|_{D=D_{n}}}+D_{n}
$$

Analysis of the final formula of this tribovibroacoustic model involves identification of two characteristic points. For time $\theta=0$, the above formula yields $D=D_{n}$, that is the amplitude of rated vibrations for a new object, whereas for:

$$
\theta=\theta_{a w}=\left(\left.\gamma \frac{d N_{N}}{d}\right|_{D=D_{n}}\right)^{-1}
$$

we have $\mathrm{D}=\infty$, which can be interpreted as a failure. Value of time to failure:

$$
\theta_{A w}=\left(\left.\frac{d N_{z}}{d D}\right|_{D=D_{n}}\right)^{-1}
$$

is inversely proportional to tribo-vibro-acoustics loss $\gamma$, and sensitivity of wear to vibrations: $d N_{z} / d D$.

The value of vibration amplitude $D$ and the rate of their changes during operation $\theta$ depend on these characteristics as well as on the total power of machine tribological processes.

Both the model and the insight into vibration feedback of wear processes it provides are very valuable. This model shows that the time of failure $\theta A w$ can be predicted for a new machine knowing only the vibration gradient of lost power:

$$
\left.\frac{d N_{z}}{d D}\right|_{\mathrm{D}=D_{n}}
$$

and its lossine TWA $\gamma$. Sequential utilization of the model for prediction of a machine state and its vibrations is possible as well $[6,9]$.

In practice however, the current state of a machine can be identified by observing the object functioning, that is, its main output of converted energy (or a product) and dissipation output where residue processes e.g. thermal, vibratory, acoustic, electromagnetic are observed.

Observation of these outputs provides a vast range of possibilities of machine diagnosis through:

*observation of operational processes by continuous monitoring their parameters or by testing the machine efficiency on special test stations (power, speed, pressure etc.),

* studying of its product quality, consistence of measurements, links, etc, as generally speaking the better the machine technical state the better the production quality,

* observation of residual processes which provide the basis for many attractive diagnostic methods based mainly on symptom-based models. 
The above presented problems should be solved with the use of the newest technologies. This is an area for wide application of computer aid and automation of procedures in the field of: holistic and symptom-based modeling, inference, planning and performance of tests, utilization of artificial intelligence encompassing counselling systems and neuron networks with fuzzy logic [7,10,19].

Knowledge and technology of diagnosis are always connected with the kind of diagnostic model that can be developed for a given object.

Reduction of destructive influence of an object physical aging and fatigue wear is necessary in all stages of its existence. Desirable effects of the number of reductions in the number of technical object failures can be achieved in:

* the field of construction - by appropriate selection of materials and their shapes to withstand the loads, shaping unit compressions, choice of adequate materials and substances for friction pairs, elimination of dry friction, wide application of appropriate tightening, providing appropriate temperature;

* in the field of technology - by choosing optimal treatment, shaping an optimal surface layer, choice of appropriate thermal and thermal-chemical treatment, proper installation and adjustments;

* in the field of operation - by complying with frequencies and scope of services (smearing, regulation, protection from corrosion), avoiding overloads and abrupt speed changes, monitoring of technical condition.

Inappropriate operation and maintenance causes intensive impact of wear processes leading to premature failures and threats to safety.

\section{Machine diagnostics throughout its life time}

Each machine undergoes four stages of its existence: validation, design, manufacturing and operation. Increasing requirements from machines provide a set of criteria which are studied in each of these stages [9,22,24]. Methods and means providing such possibilities of control of quality of machines - fulfilment of these criteria - are the included in the field of technical diagnostics.

Technical diagnostics covers the following activities: assessment of the state, genesis and prognosis of the state. These activities are performed by intelligent and often automated diagnostic systems (mobile software and hardware with self-learning loop and risk assessment). The set of diagnostic parameters is identified from a set of input parameters accompanying the machine operation. Determination of a set of damage sensitive diagnostic parameters should take into consideration:

- ability of reflect the machine state changes in time during its operation and maintenance.

- amount of information on technical state of the transmission,

- sensitivity to parameters value during operation.

An advantage of the above presented methods is that they provide the possibility to choose, from a set of output parameters, both the one-element and multi - element sets of diagnostic parameters.

Criteria for optimization of the set of parameters include:

- $\quad$ parameters should characterize the destruction process and be closely related to it;

- parameters should be sensitive to changes in the object degradation process;

- the number of diagnostic parameters cannot be too big as too many of them make it difficult to identify the process of devastation and sometimes it becomes even impossible;

- diagnostic parameters should be measurable;

- the must exists reliable statistical and analytical data of the distinguished parameters. 
The diagnostic system becomes a subject of separate considerations and diagnostic features of such systems require elaboration and description (with mathematical formalization). A diagnostic system is a set of diagnostic agents, set of methods and means for obtainment, processing, presentation, generation and collecting information and a set of engineering objects, their models and algorithms for diagnostics, generation and prognosis of states as well as relations between these elements. It is intended to support making reliable decisions on an object's belonging to a respective class of states. The structure of a diagnostic system proposed in fig. 6 shows the basic relations between the test object and its diagnostic model as well as the system of its state identification and decision making.

Diagnostic systems belong to a class of automated computer systems and are characterized by:

- the goal of their operation is identification of the state other objects, actually they do not affect this state,

- this goal involves generating a diagnose on the object properties into information on its state.

For these reasons, the attention must be focused on the informative structure of the diagnostic system and it needs to be designed, optimized and evaluated in terms of the information flow possibility. Different forms of components and their different functions provide the possibility of creation of diagnostic systems with different structures and with diversified automation degree.

The process of a machine handling is as follows:

a) for a usable machine: testing the state - performance of necessary servicing activities -prognosis of the machine state - scheduling the next survey;

b) for an unusable machine: assessment of the machine state - finding causes of its failures (maladjustment, damage, poor quality of repairs or liquidation) - removal of failure - assessment of a repair quality - performance of servicing activities - prognosis of the state - date of the next diagnosis.

Problems connected with diagnostics are usually addressed by a diagnostic team. They define the scope, methods and means necessary to supervise machine operation mad maintenance (critical, important, unimportant). The tasks are performed in cooperation with the departments of the chief mechanic, production supervisor and manager of marketing department and accountancy department. Particular departments of a company are interested in different information items on the condition of machines, depending on the specificity machine use and production.

\section{Examples of practical diagnosis of the state}

The machine technical state prognosis process can be carried out by different methods $[5,7,14]$ : forecasted symptom value, times of a machine operation or other prognoses the states of machine (e.g. extrapolation trend methods and adaptive methods) [16,20].

The selected problems of diagnosing the state of machines using forecasting methods have been validated in several different studies on operation and maintenance, and some examples of the issues and their solutions are presented selectively below.

Schematic diagram of the predictive diagnosis consists of: selection of the optimal set of diagnostic parameters, choice of the optimal method of forecasting, and assessment of predictive quality of the diagnosis. The optimal set of diagnostic parameters guarantees:

1. the minimum number of points needed to run the time prediction;

2. changes in units of the machine during operation resulting from replacement or adjustment of teams and variable operating conditions (road conditions, climate, quality of handling) that cause abrupt changes in the value of diagnostic parameters;

3. the maximum value of the time step;

4. the cardinality of the set of relevant parameters to the application. 
The choice of a forecasting method is conditioned by: the forecast horizon; the minimum number of points needed to run the time prediction; the number of measurements before time $t_{b}$ to calculate the predictive diagnosis; the variable degradation bands, causing abrupt changes in symptoms. Evaluation of the quality of diagnosis prognostic prediction error is determined.

Studies of the procedures of machine prognosis state in the aspect of determining methods for forecasting damage, examining the influence of horizon prognoses in the recognition of damage, and studies of the influence of the number of diagnostic parameters on the damage prognosis, were carried out on the basis of: determination of a set of prognosis methods for values of diagnostic parameters, and prediction of the next time of diagnosis - according to the process presented in [25].

Summing up the research performed for the state prognosis method, it can be stated that:

$>$ considering low values of the curvilinear correlation coefficient $(<0.8$ ), high values of prognosis of damage, and negative values for the next operation dates of the objects examined in analytical models (linear model, exponential model, model Brown-Mayer, and Holt model), for potential applications it is necessary to use the Brown-Mayer model and the Holt model;

$>$ the accepted optimization criteria, and the presented algorithm identify unambiguously the prognosis methods and the method of estimating the next diagnosis term. This confirms the appropriateness of the formulated procedure and will provide the basis for methodology to be used for the machine state recognition and in the field of state prognosis for other objects.

The analysis of results of the research on the machine state prognosis methodology allows of formulate conclusions in the areas of: diagnostic parameters optimization and state prognosis. After appropriate verification the presented conclusion can provide the basis for application of dedicated software for a machine state recognition system in an online (board system) and off-line (for a stationary system) modes.

\section{Determining the next term of diagnostics}

Evaluation of state changes based on change monitoring of diagnostic parameters $\left(\Theta_{\mathrm{b} 1}\right.$ determination) was carried out for a vehicles STAR 200, for the results of tests of peripheral clearance of the main shaft - shown in Table 2.

This procedure uses the already approved method of determining the limit value of the symptom $[8,22]$ :

$$
S_{j g r}=-\bar{s}+\sigma_{s} \sqrt{\frac{P_{g}}{2 A}}
$$

where: $P_{g}$ - repair policy of the works, $A=k\left(1-P_{g}\right), k=\{1-9\}$.

The presented relationship allows determine the term of the next diagnostics in relation (11) to the current value of a measured symptom, its relation to the value symptoms of limiting with considering the repair policy of the works [22]:

$$
\Theta_{b 1}=\frac{\left(1-P_{r}\right)\left(S_{g r}-S_{j}\right)}{S_{j}}
$$

Determination of this value (12) based on the value of data on a circumferential backlash of the main shaft of $\mathrm{t}$ STAR 200 vehicle in operation is shown in Table 1. 
Table 1. Measuring data of peripheral clearance of examined main shaft.

\begin{tabular}{|l|l|l|l|l|l|l|l|l|l|r|}
\hline Clearance- $\mathrm{S}_{\mathrm{d}}[\mathrm{mm}]$ & 6,6 & 7,5 & 8,1 & 9,5 & 10,5 & 14,2 & 15,7 & 16,5 & 16,9 & $\begin{array}{r}17, \\
1\end{array}$ \\
\hline Mileage $\Theta_{\mathrm{j}}\left[\mathrm{x} 10^{3} \mathrm{~km}\right]$ & 2 & 24 & 48 & 72 & 96 & 120 & 144 & 168 & 192 & 216 \\
\hline
\end{tabular}

In accordance with relations (11) $[7,10,15]$ it was evaluated: $\mathbf{S}_{\mathbf{j g r}}=\mathbf{1 8 , 9 9}$, accepted in (17) $\mathrm{k}=4, \mathrm{P}_{\mathrm{g}}=0,95, \mathrm{~A}=\mathrm{k}\left(1-\mathrm{P}_{\mathrm{g}}\right)=0,2$. Evaluating further values $\Theta_{\mathrm{b} 1}$ according to (12) for accessible measuring data (Table 2) there was fixed a trend of transmission gear clearance changes (state symptom) in the following form:

$$
\mathrm{S}_{\mathrm{j}}=6,0091 e^{0,1189 \Theta_{j}}
$$

Thus, in table 2 there are presented values $S^{*}{ }_{j}$ (calculated according to (13) for determined mileages $\Theta_{j}$, which allowed to determine forecast error $\mathrm{e}^{*}$, defined as [24]:

$$
\mathrm{e}^{*_{\mathrm{p}}}=\left(\mathrm{S} *_{\mathrm{j}}-\mathrm{S}_{\mathrm{j}}\right) / \mathrm{S} *_{\mathrm{j}}
$$

Table 2. Calculations of results.

\begin{tabular}{|c|c|c|c|c|c|c|c|}
\hline $\begin{array}{c}\text { Mileage } \Theta_{\mathrm{j}}\left[10^{3}\right. \\
\mathrm{km}]\end{array}$ & 2 & 24 & 48 & 72 & 96 & 120 & 144 \\
\hline $\mathrm{S}_{\mathrm{d}}$ & 6,6 & 7,5 & 8,1 & 9,5 & 10,5 & 14,2 & 15,7 \\
\hline$\Theta_{\mathrm{b} \underline{ }[\mathrm{km}]}$ & 488 & 4560 & 7776 & 7776 & 7644 & 720 & Reversal of sign \\
\hline $\mathrm{S}_{\mathrm{i}}^{*}$ & 7,27 & 9,75 & 10,4 & 13,19 & 11,16 & 11,36 & $\mathrm{X}$ \\
\hline $\mathrm{e}_{\mathrm{p}}^{*}[\%]$ & 10 & 30 & 29 & 37 & 6 & 20 & $\mathrm{X}$ \\
\hline
\end{tabular}

The average forecast error of a symptom value $\left(S_{j}\right)$ for mileages $Q_{j}$ accepted in the measurements is $20 \%$. A relatively big forecast error of the proposed method may be decreased by optimising activities undertaken in measuring accessible data from servicing. The performed analytical studies, testing conformity of the measured values of symptoms for the determined mileages (table 2) by means of the proposed methods [22], allow of propose the following actions:

- $\mathrm{S}_{\mathrm{jgr}}$ should be maximised in the procedure of step-by-step determination by periodical changes of $\mathrm{P}_{\mathrm{g}}$ and $\mathrm{k}$ [22];

- good approximations are obtained for an increase of $\mathrm{P}_{\mathrm{g}}$, and $\mathrm{k}$ in a half of the vehicle operational time (according to data from table 2).

For $\mathrm{S}_{\mathrm{jgr}}$ accepted from standard (or from operator's manual), results of theoretical calculations are well represented by operational data:

- approximation of $\Theta_{j}$ evaluated from operational data is satisfactory for relation (12);

- presentation of frequency the examination with registrations of exponential trend of the examined symptoms.

The results of the experiments allow to show a way of reasoning and inference in terms of the future diagnostic agent, an intelligent system monitoring critical technical systems. On fig. 7 shows the general idea of an agent system to be used for diagnosis and observation of critical systems as well as for information flow handling in the future of diagnostics. We can also see a need to provide some other information concerning the system operation including: load, current time of system life, and also some previous records on the system history contained in the maintenance data base.

The last step of SAD is to develop a measure of further operation risk and make other decision of service type / repair resulting from the assessment of the status and information about the object and the environment. Thus, it can be implemented, as shown graphically, 
and as you can see, for innovative objects which lack experience in operation and have no operating history there is only one chance to fulfill this mission by using a self-learning mode.

\section{Conlusions}

Apart from tribology, reliability, security theory and the theory of operation technical diagnostics is one of the fundamental issues connected with rational operation and maintenance of objects. Understanding the physical phenomena occurring during the operation of the machine allows determine of the qualitative relationships taking place between destructive processes and the machine state. The growing demand for diagnostics goes hand-in-hand with applications of new inventions in microelectronics, computer technology, fractal theory, neural networks and artificial intelligence - effectively support diagnostics capabilities.

The basic condition for these considerations to achieve success is to establish a uniform wear of machinery or provide knowledge of the measured symptoms trend (knowledge of intensity of damage). The phenomena that cause wear of assemblies are very complex and their formation is usually affected by several factors, so including them all in the forecasting process is impossible.

The issue of machine tests for the process of prognosis to be used for estimation of the technical state, as well as legal acts related to safety of the user and the environmental protection, are a stimulus to search for new prognostic methods, determining new measures and tools that can describe the current state in the machines specific work condition. This paper attempts to summarize basis mathematical rules to be used for machine condition forecasting based on multi-symptom prognostic methods.

The presentation of the procedures for machine state prognosis allows of determine the parameters, according to the accepted criteria: diagnostic parameters set, diagnostic parameters values prognosis and machine operation date estimation.

\section{References}

1. R. Ahmad, S. Kamarudin, An overview of time-based and condition - based maintenance in industrial application. Computers \& Industrial Engineering (2012)..

2. I. Birger, Technical diagnostics. Nauka, Moscov (1978).

3. C. Cempel, Basics of vibroacoustic diagnostics of machines. WNT, Warsaw (1982).

4. G. Box, G. Jenkins, Time series analysis, forecasting and control, London (1970).

5. L. Bowerman, R.T. O'Connel, Forecasting and Time Series. Doxbury Press, USA (1979).

6. C. Cempel, H.G. Natke, An introduction to the holistic dynamics of operating systems. Progress Report, No.2 (1996).

7. C. Cempel, M. Tabaszewski, Multidimensional condition monitoring of machines in nonstationary operation. Mechanical Systems and Signal Processing (21), (2007).

8. N.R. Draper, H. Smith, Regression analysis of used. BNInż., Warsaw (1973).

9. P. Eykhoff, Identification in dynamical systems, BNInż., Warsaw(1980).

10. W. Findeisen, System analysis - base and methodology. PWN Warsaw (1985).

11. K. Mańczak, Methods for identifying the multidimensional of control objects. WNT Warsaw (1971).

12. S. Niziński, R. Michalski, Diagnosis of technical objects. ITE Radom (2002).

13. T. Uhl, J. Giergiel, Identification of mechanical systems. PWN Warsaw (1990).

14. A.K.S. Jardine, D. Lin, D. Banjevic, A review on machinery diagnostics and prognostics implementing condition-based maintenance, Mechanical Systems and Signal Processing, 20 (2006). 
15. J. Luo, M. Namburu, K. Pattipati, L. Qiao, M. Kawamoto, Model-based prognostic techniques, in: Proceedings of the IEEE. Systems Readiness Technology Conference ( 2003).

16. Ł. Muślewski, Study and assessment of transport system operation efficiency. Journal of KONES Powertrain and Transport, Vol. 17, No. 4, Warsaw (2010).

17. V.T. Tran, B.S. Yang, M.S. Oh, Machine condition prognosis based on regression trees and one-step-ahead prediction. Mechanical Systems and Signal Processing, 22, (2008).

18. J. Qu, M.J. Zuo, An LSSVR - based algorithm for online system condition prognostics. Expert Systems with Applications, 39 (2) (2012).

19. H. Tylicki, B. Żółtowski, Determination methods of the next diagnosis term of transport vehicle. Archives of Transport, Warsaw (2001).

20. A. Zeliaś, Prognosis theory. PWE Warsaw (1984).

21. B. Żółtowski, The methods of virtual engineering in the research risks status, safety and environmental operated machines. UTP Bydgoszcz (2012).

22. B. Żółtowski, The study of environmental risks losing the suitability of technical systems. UTP Bydgoszcz (2013).

23. M. Żółtowski, Management information systems in engineering and manufacturing. ITE - PIB, Radom (2011).

24. M. Żółtowski, Investigations of harbour brick structures by using operational modal analysis. Polish Maritime Research, No. 1/ (81), vol.21 (2014).

25. M. Żółtowski, Assessment State of Masonry Components Degradation. Applied Mechanics and Materials Vol. 617 (2014).

26. B. Żółtowski, M. Żółtowski, Vibrations in the Assessment of Construction State. Applied Mechanics and Materials Vol. 617 (2014). 